\title{
Orientation Dependence of Hardness and Reduced Modulus of Single Crystal Sapphire Surface Measured by Nanoindentation
}

\author{
Toshiro Okawa ${ }^{1,2, \text { * }, \text { Ian Thomas Clark }}{ }^{3}$, Katsuhiko Tashiro ${ }^{2,4}$, Hideo Honma ${ }^{2,4}$, \\ Kazuhiro Yoshihara ${ }^{5}$, Osamu Takai ${ }^{2,4}$ \\ ${ }^{1}$ Scienta Omicron, Inc., Tokyo, Japan \\ ${ }^{2}$ Materials \& Surface Engineering Research Institute, Kanto Gakuin University, Kanagawa, Japan \\ ${ }^{3}$ Seiko Epson Corporation, Nagano, Japan \\ ${ }^{4}$ Research Advancement and Management Organization, Kanto Gakuin University, Kanagawa, Japan \\ ${ }^{5}$ National Institute for Material Science, Ibaraki, Japan \\ Email address: \\ Toshiro.Okawa@scientaomicron.com (T. Okawa) \\ *Corresponding author
}

\section{To cite this article:}

Toshiro Okawa, Ian Thomas Clark, Katsuhiko Tashiro, Hideo Honma, Kazuhiro Yoshihara, Osamu Takai. Orientation Dependence of Hardness and Reduced Modulus of Single Crystal Sapphire Surface Measured by Nanoindentation. International Journal of Materials Science and Applications. Vol. 8, No. 5, 2019, pp. 76-80. doi: 10.11648/j.ijmsa.20190805.11

Received: September 15, 2019; Accepted: September 26, 2019; Published: October 10, 2019

\begin{abstract}
Nowadays, industrial products are downsized, and the structure of materials is controlled with the nanometer precision, and it becomes very important to measure the mechanical properties of local area of bulk material. Especially the hardness and the elastic modulus are important mechanical properties. The orientation dependence of hardness and reduced modulus of single crystal sapphire surface was investigated by nanoindentation. The conventional technique to measure the hardness of materials using an optical micrometer cannot evaluate mechanical properties of a local region of several $\mu \mathrm{m}$ or less. However, nanoindentation can measure mechanical properties of very small surface area of materials, and is expected to detect the micro structure dependence of mechanical properties. Nanoindentation uses very small indenter made of diamond, and measures the indentation depth. The measured depth is converted to the indented area size using the area function. The area function of the indenter can be obtained using a standard material (fused quartz) in advance. Therefore nanoindentation can measure the indented area size without using an optical micrometer. In this report, it was shown that the nanoindentation could detect the structure dependence of mechanical properties of materials. The specimen was a single crystal sapphire with c - axis surface, and the indenter was Berkovich type diamond tip. It was confirmed that the nanoindentation hardness was the lowest, and the reduced modulus was the largest, when the ridge line of indenter was oriented to the $\mathrm{m}$ - axis of single crystal. The nanoindentation could detect the structure dependence of a local area of mechanical properties materials.
\end{abstract}

Keywords: Nanoindentation, Hardness, Elastic Modulus, Single Crystal, Sapphire

\section{Introduction}

Nowadays, industrial products are downsized, and the structure of materials is controlled with the nanometer precision. The nanometer scale thin film technology is not only for semiconductor devices, but also applied to the surface finishing technology such as plating. Therefore, it becomes very important to measure the mechanical properties of local area of bulk material. Especially the hardness and the elastic modulus are important mechanical properties of a local area of bulk material. The conventional technique to measure the hardness of material is the quasi-static indentation test. However, this test needs an optical microscope to measure the indented area size. Therefore, mechanical properties of a local region of several $\mu \mathrm{m}$ or less cannot be evaluated. In 1992, W. C. Oliver and G. M. Pharr proposed the depth sensing 
indentation method [1]. This method can calculate the hardness and the elastic modulus by measuring the indentation depth. They proposed a new function (area function) to convert the indentation depth into the indented area size without using an optical microscope. This method was standardized as ISO 14577 in 2002, and is now widely used as a nanoindentation method. The most commonly used indenter in this method is a diamond Berkovich [2] type which is a three-sided pyramid and has the total included angle of 142.3 degrees and a face angle of 65.27 degrees. A Berkovich indenter is designed to have the same ratio of projected area to indentation depth as that of a Vickers indenter. Therefore, the nanoindentation hardness obtained by a Berkovich indenter can be compared with the Vickers hardness.

The hardness and the elastic modulus at a local area are expected to be greatly influenced by the crystal orientation or the presence of microdefects of specimens. However, the conventional method using an optical microscope cannot divide the effect of these microstructures in a specimen from the obtained measurement results. The nanoindentation method has a very small measurement area. Therefore, it is expected to be able to measure the effect of the crystal orientation or microdefects of specimens on the mechanical properties of a material [3-7]. In this report, in order to investigate the effect of the crystal orientation, the hardness and the reduced modulus of the single crystal of sapphire were measured by changing the direction angle of Berkovich indenter.

\section{Experimental}

The hardness $(H)$ is obtained by dividing the indentation load $(P)$ by the indented area $(A)$. In the nanoindentation method, the relationship between the projected indented area $\left(A_{c}\right)$ and the indentation depth $(h)$ is called the area function $\left(A_{c}(h)\right)$ which is obtained in advance. When an indenter indents a specimen, the vicinity of the specimen surface is elastically deformed as shown in Figure 1, and two kinds of indentation depth are defined. One is the maximum depth of indented area $\left(h_{\max }\right)$, and the other is the contact depth $\left(h_{c}\right)$.

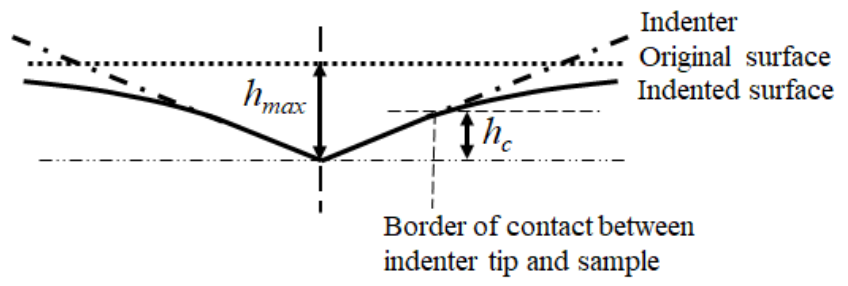

Figure 1. The cross-section diagram of contact between indenter and specimen.

The contact depth can be obtained by the maximum depth subtracting the amount of deflection due to the elastic deformation. Oliver et al. proposed the procedure to get the contact depth by Sneddon's theory of elasticity [8], and derived the following equation) using shape factor of the indenter $(\varepsilon=0.75[1])$ and the relationship between load and indentation depth.

$$
h_{c}=h_{\max }-\varepsilon \frac{s}{P}
$$

$S$ is the stiffness defined as the derivative value of indentation load at the maximum depth $\left(\left.\mathrm{S} \equiv \frac{d P}{d h}\right|_{h_{\text {max }}}\right)$.

Nanoindentation hardness is obtained from the maximum indentation load $\left(P_{\max }\right)$ divided by the indented area. The contact depth $\left(h_{c}\right)$ is calculated from eq. 1 , and the indentation area is obtained from the area function $\left(A_{c}\left(h_{c}\right)\right)$. Using these values, the nanoindentation hardness $\left(H_{I T}\right)$ and the reduced modulus $\left(E_{r}\right)$ can be obtained using eq. 2 and eq. 3 respectively.

$$
\begin{aligned}
& \mathrm{H}_{I T}=\frac{P_{\max }}{A_{c}\left(h_{c}\right)} \\
& \mathrm{E}_{r}=\frac{S \sqrt{\pi}}{2 \sqrt{A_{c}\left(h_{c}\right)}}
\end{aligned}
$$

The reduced modulus is the combination of the elastic modulus of the specimen and that of the indenter material as indicated by eq.4. Here, $E_{\text {specimen }}$ is the elastic modulus of specimen, and $v_{\text {specimen }}$ is the Poisson's ratio of specimen. $E_{t i p}$ is the elastic modulus of indenter material, and $v_{t i p}$ is the Poisson's ratio of indenter material. The material of the indenter is diamond, and $E_{t i p}$ is 1070 [GPa] and $v_{\text {tip }}$ is 0.07 [9].

$$
\frac{1}{E_{r}}=\frac{1-v_{\text {specimen }}^{2}}{E_{\text {specimen }}}+\frac{1-v_{t i p}^{2}}{E_{\text {tip }}}
$$

In this experiment, Tribo Indenter manufactured by Hysitron was used. The area function of Berkovich type indenter used in this experiment was obtained by performing an indentation test on a fused silica. The reduced modulus of fused silica has been already determined by Hysitron Inc. as 69,6 GPa. Using the contact depth and stiffness obtained from the load - displacement curve, the area function of Berkovich type indenter was calculated based on eq.3. The area function of the Berkovich indenter used in this experiment is shown in Figure 2.

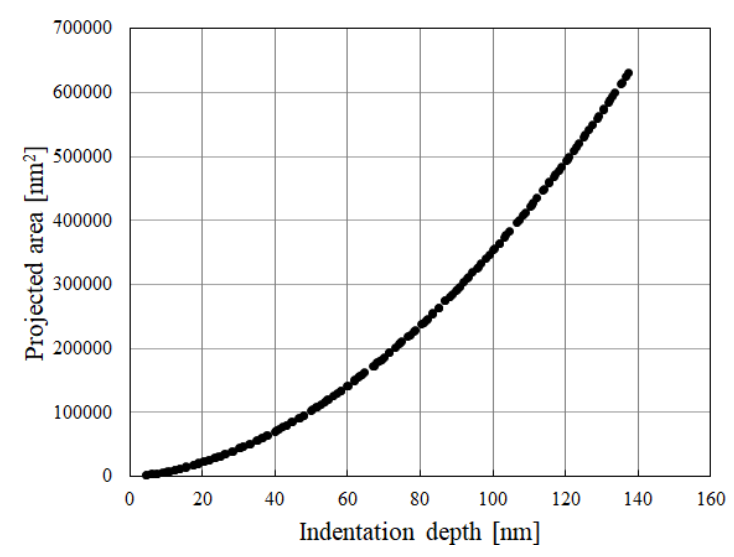

Figure 2. The change of projected area of indentation with the indentation depth.

The ordinate is the projected area of indentation $\left[\mathrm{nm}^{2}\right]$, and the abscissa is the indentation depth [nm]. However, it has been reported that the tip shape of the indenter does not show a triangular pyramid shape when the contact depth is smaller than $25 \mathrm{~nm}$ (=40nm; indentation depth), but is a sphere shape [10]. 


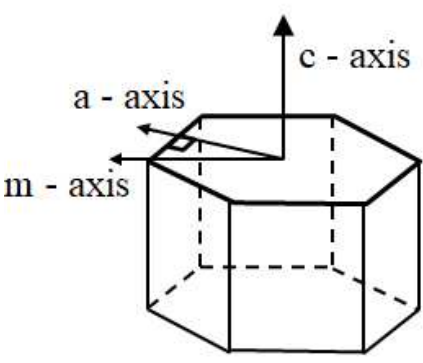

(a)

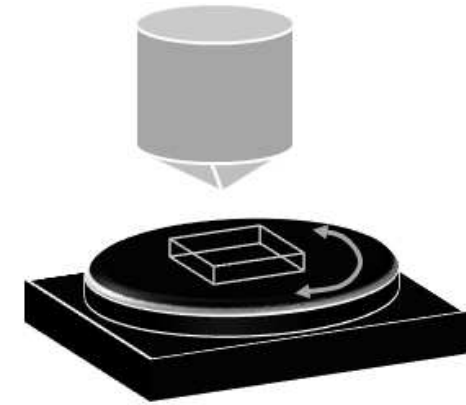

(b)

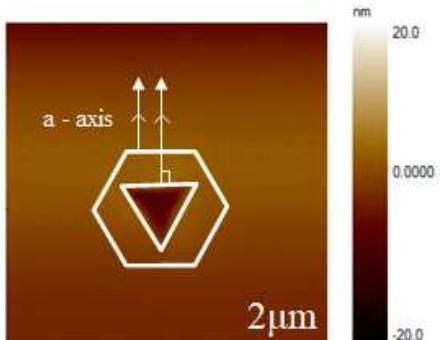

(c)

Figure 3. (a) Schematic diagram of crystal orientation of hexagonal crystal, (b) Schematic diagram of indenter and sample stage, (c) Surface image (2 $\mu$ m square) of indented area (load was $3 \mathrm{mN}$, and rotation angle of the indenter was $0^{\circ}$ ).

The single crystal sapphire having a c - axis surface was used as a specimen. The single crystal sapphire has a hexagonal crystal structure schematically shown in Figure 3 (a). As shown in Figure 3 (a), the hexagonal crystal is 6-hold rotational symmetry with respect to c - axis. In general, two directions on the single crystal sapphire surface are defined. One is $\mathrm{m}$ - axis directed to the apex of hexagonal, and the other is a - axis directed to the side of hexagonal. The $\mathrm{m}$ - axis of specimen was confirmed by XRD. As shown in Figure 3 (b), the sample stage used was a turntable that can rotate around $\mathrm{c}$ axis horizontally with respect to the indentation direction. The specimen was set to this sample stage using surface roughness measurement apparatus attached to the nanoindentation system. As shown in Figure 3 (c), the side of the indenter was parallel to a - axis by rotating sample stage, and this rotation angle of the indenter was defined as $0^{\circ}$.

The indentation conditions were as follows; the maximum load was $3[\mathrm{mN}]$, the loading - unloading velocity was 600 $[\mu \mathrm{N} / \mathrm{s}]$, and the holding time was $1[\mathrm{~s}]$. The turntable was rotated from $0^{\circ}$ to $60^{\circ}$, and the rotation angle was changed every $10^{\circ}$. The nanoindentation hardness $\left(H_{I T}\right)$ and reduced modulus $\left(E_{r}\right)$ were measured at 25 points on the specimen at each rotation angle.

\section{Results and Discussion}

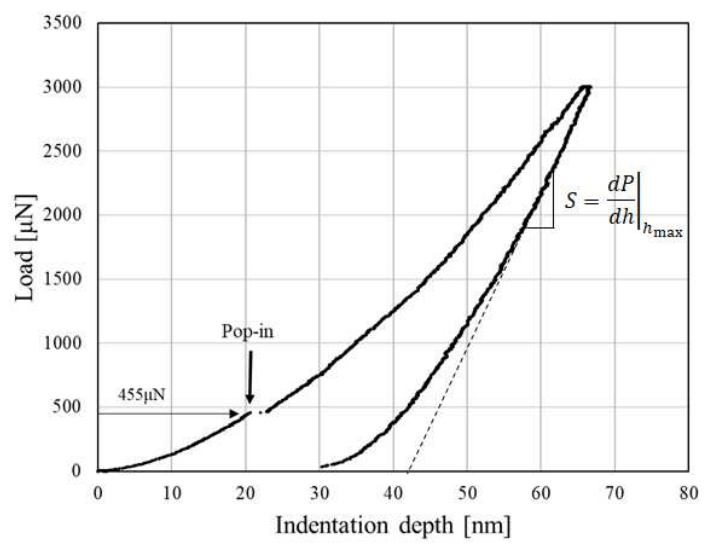

Figure 4. The load-displacement curve at the rotation angle of $0^{\circ}$.

Figure 4 shows the load - displacement curve when the indentation test was performed at the rotation angle of $0^{\circ}$. The ordinate represents the indentation load $[\mu \mathrm{N}]$, and the abscissa represents the indentation depth [nm]. When the load is 455 $\mu \mathrm{N}$, a pop-in phenomenon that the displacement increases without increasing the load was observed. The cause of the pop-in phenomenon is thought to be the high-speed propagation of crystal dislocations generated by the load. These dislocations cause plastic deformation instantaneously, and increase displacements [11-12]. This pop-in phenomenon could be observed on the load - displacement curve at every rotation angle.

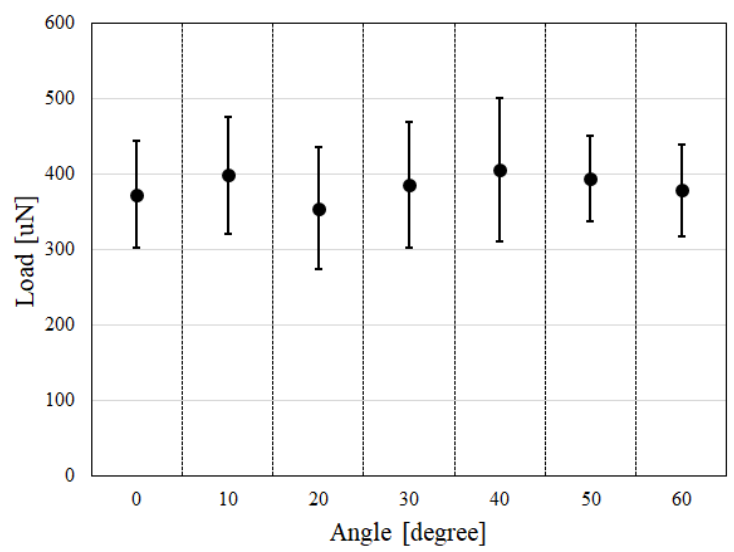

Figure 5. The relation between the load when pop-in phenomenon was observed and the rotation angle of indenter.

Figure 5 shows the relation between the load when pop-in phenomenon was observed and the rotation angle. The load when pop-in was observed was independent of the rotation angle. The indentation depth when the pop-in phenomenon occurred was about $20 \mathrm{~nm}$ (contact depth of $12 \mathrm{~nm}$ ) at any rotation angle. When the indentation depth is less than $40 \mathrm{~nm}$, it is considered that the tip shape of the indenter is not an ideal triangular pyramid shape, but a spherical shape [5]. In other words, when the indenter initially contacted the specimen, the specimen was indented with the spherical indenter, and the stress on the specimen is uniformly transmitted independent of the rotation angle. Therefore, it was considered that the pop-in phenomenon was independent of the rotation angle of the specimen in the range of the indentation depth where the shape of the indenter could be regarded as axisymmetric. 


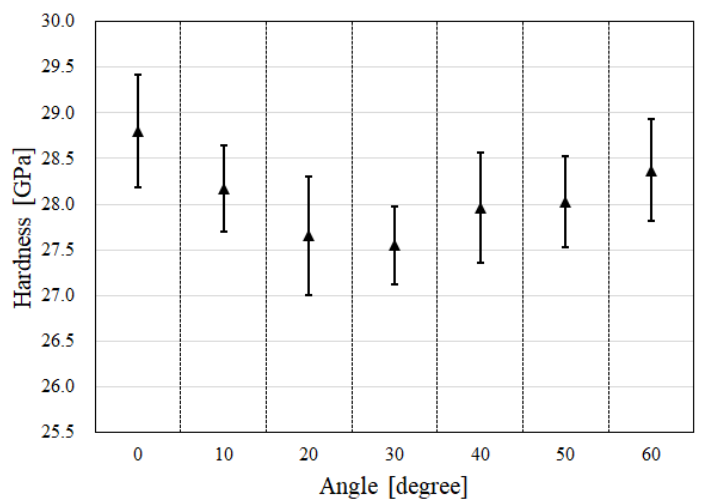

Figure 6. The relation between the nanoindentation hardness and the rotation angle of indenter.

The relationship between nanoindentation hardness and the rotation angle is shown in Figure 6, and that between the reduced modulus and the rotation angle is shown in Figure 7. The indentation load was $3 \mathrm{mN}$. Nanoindentation hardness $\left(H_{I T}\right)$ decreased as the rotation angle changed from $0^{\circ}$ to $30^{\circ}$, then increased as the rotation angle changed from $30^{\circ}$ to $60^{\circ}$. The lowest nanoindentation hardness was $27.5 \mathrm{GPa}$, and the highest was $28.8 \mathrm{GPa}$.

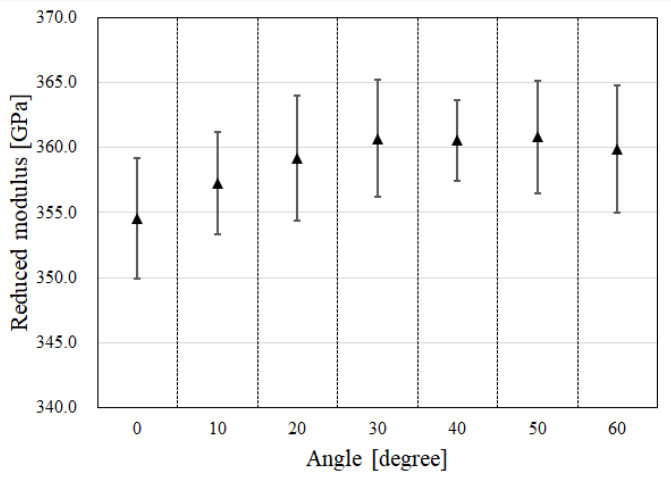

Figure 7. The relation between the reduced modulus and the rotation angle of indenter.

The reduced modulus increases as the rotation angle changed from $0^{\circ}$ to $30^{\circ}$ and then slightly decreased as the rotation angle changed from $30^{\circ}$ to $60^{\circ}$. The largest reduced modulus was $361 \mathrm{GPa}$, and the smallest was $355 \mathrm{GPa}$. In this case indentation depth was larger than $60 \mathrm{~nm}$ and the shape of indenter was the triangular pyramid as shown in Figure 3 (c).

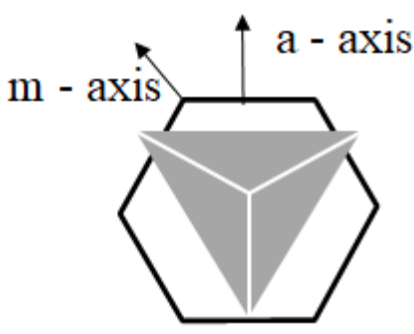

(a)

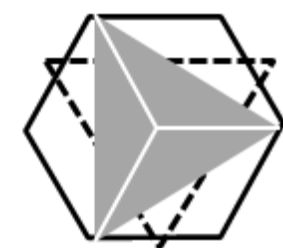

(b)
Figure 8. The relationship between the crystal direction and the rotation angle of indenter, (a) rotation angle: $0^{\circ}$, (b) rotation angle: $30^{\circ}$.
Figure 8 shows a schematic illustration of the crystal orientation of specimen and the rotation of indenter. Figure 8 (a) shows the ridge line of the tip was oriented to a - axis that means rotation angle is 0 , and Figure 8 (b) shows the ridge line of the tip was oriented to $\mathrm{m}$ - axis, and the rotation angle is $30^{\circ}$. Since the shape of Berkovich indenter is a triangular pyramid, the three ridges of the trihedron are spaced at regular intervals of 120 degrees. Because of the symmetry relation between the indenter and the sapphire crystal, the indentation measurements at rotation angles of $40^{\circ}, 50$, and 60 were considered to be equivalent to indentation tests at $20^{\circ}, 10^{\circ}$, and $0^{\circ}$ respectively. Therefore. it is concluded that the hardness along $\mathrm{m}$ - axis were low and the reduced modulus was large along $\mathrm{m}$ - axis.

$\mathrm{J}$. W. Fischer et al. reported that the $\mathrm{m}$ - axis of sapphire was more fragile than the a-axis [13]. This reported result was coincided with these experimental results. When the indenter was indented to enough depth of the specimen, the stress is transmitted from the apex of indenter to the specimen. It was reported that the stress was concentrated along the ridge line of the indenter [14]. Therefore, if the ridge line of indenter was oriented to $m$ - axis, the stress was concentrated to the fragile $\mathrm{m}$ - axis, and eventually the hardness along $\mathrm{m}$ - axis became low. On the other hand, F. A. Strobel reported that the elastic modulus of sapphire was independent of the crystal orientation [15]. However, in this experiment, the reduced modulus depended on the rotation angle, and was large at a rotation angle of $30^{\circ}$. To derive the elastic modulus from the reduced modulus, Poisson's ratio must be known. However, there has been no report on the crystal orientation of Poisson's ratio of sapphire and could not be compared directly with the results given by F. A. Strobel.

\section{Conclusion}

The orientation dependences of hardness and reduced modulus of single crystal sapphire surface were measured by nanoindentation. Both nanoindentation hardness and reduced modulus were shown to have crystal orientation dependence. When the indenter initially contacted specimen, the apex shape of the indenter was regarded as spherically symmetry, and the stress was uniformly transmitted in all directions and the fracture behavior was independent of crystal orientation. When the indenter was indented to enough depth, the stress was transmitted from the apex of indenter to the sample. It was shown that the nanoindentation hardness was low, and the reduced modulus was large, when the ridge line of the indenter is oriented to the $m$ - axis. It was indicated that the nanoindentation technique could detect the structure dependence of mechanical properties materials. The evaluation of hardness and reduced modulus using the nanoindentation technique is expected to cope with the increasing demand for nanostructure materials. 


\section{References}

[1] W. C. Oliver and G. M. Pharr, J. Mater. Res., 7, 1564 (1992).

[2] M. M. Khrushchov \& E. S. Berkovich, Ind. Dia. Rev., 11, 42 (1951).

[3] Hu Huang, Jiwang Yan and Scr. Mater., 102, 35 (2015).

[4] Tama's Csana'di, Marek Bl'anda, Nguyen Q. Chinh, Pavol Hvizdos and Ja'n Dusza., Acta Mater., 84, 397 (2015).

[5] Hidetoshi Somekawa, Tomohito Tsuru, Alok Singh, Seiji Miura and Christopher A. Schuh., Acta Mater., 139, 21 (2017).

[6] E. Renner, Y. Gaillard, F. Richard, F. Amiot and P. Delobelle., Int. J. Plasti., 77, 118 (2016).

[7] Saurav Goel, Graham Cross, Alexander Stukowski, Ernst Gamsjäger, Ben Beake and Anupam Agrawal, Comput. Mater. Sci., 152, 196 (2018).

[8] Ian N. Snedon, Int. J. Eng. Sci., 3, 47 (1965).

[9] Anthony C. Fisher-Cripps, "Nanoindentation”, Springer., 257 (2011). 3 .

[10] M. R. VanLandingham, T. F. Juliano and M J Hagon, Meas. Sci. Technol., 16, 2173 (2005).

[11] A. M. Minor, E. T. Lilleodden, E. A. Stachand J. W. Morris., J. Mater. Res., 19, 176 (2004).

[12] Payel Maiti, Ammar Eqbal, Manjima Bhattacharya, Pradip Sekhar Das, Jiten Ghosh, Anoop Kumar Mukhopadhyay, Ceram. Int., 45 (7), 8204 (2019).

[13] Johan E. Fischer, W. R. Compion, Nancy A. Jaeger and Daniel C. Harris., SPIE. 1326, 11 (1990).

[14] Ningchang Wang, Feng Jiang, Xipeng Xu and Xizhao Lu., Crystals., 8 (1), 3 (2018).

[15] F. A. Strobel, Naval Weapons Center Technical Publication 6539 (1985). 